the total metabolic equivalent (MET) to incorporate the frequency, duration and intensity by different type of activities. Minutes spent in each level of activity (e.g., sedentary, light, moderate, and vigorous) were then summed for each week. Adjusted multivariable linear models estimated the relationship between physical activity levels and disease status.

Results: Overall, nearly $80 \%$ of the study sample had predominantly peripheral spondyloarthritis. The average age for participants with peripheral disease was 53.2 (standard deviation (SD):12.9) and for those with axial disease was 44.2 (SD: 12.4). Most of the study participants were men, white, non-Hispanic, married, and had attended at least some college, regardless of predominant joint distribution (peripheral versus axial). While the median for general attitude towards regular exercise in participants with peripheral disease was 88.5, the median score was 81.0 in participants with axial disease. The median score regarding benefits of exercise/physical activity to improve general function was 97.0 among participants with peripheral disease and 95.5 among those with axial disease; the median score for pain relief was 67.0 in participants with peripheral disease and 66.5 for those with axial disease. Regardless of predominant joint distribution, walking, bicycling, and swimming were the most common types of exercise. Compared to participants with predominately peripheral disease, participants with axial disease spent more time per week engaging in light physical activities (adjusted $\beta$ : $13.0 \mathrm{hrs} /$ week; 95\% confidence interval: 0.4 to $25.6 \mathrm{hrs} /$ week) after adjusting for sociodemographic and clinical factors.

Conclusion: Patients with spondyloarthritis have positive attitudes towards physical activity/exercise, regardless of their predominant joint distribution, and believe that these activities improve general function and, to a lesser extent, relieve pain. However, patients with axial disease spend more time per week engaging in light physical activities than do those with peripheral disease.

Acknowledgement: This work was supported by a generous donation from Timothy S. and Elaine L. Peterson.

Disclosure of Interests: Shao-Hsien Liu Grant/research support from: Novartis, Divya Shridharmurthy: None declared, Kate Lapane Grant/ research support from: Novartis, Merck, Pfizer, Janssen, Consultant for: Pfizer, Stephen Morais: None declared, Catherine Dubé Grant/research support from: Novartis, Jonathan Kay Grant/research support from: Gilead Sciences, Pfizer, UCB Pharma, Consultant for: AbbVie, Boehringer Ingelheim $\mathrm{GmbH}$, Celltrion Healthcare, Merck Sharp \& Dohme Corp., Novartis Pharmaceuticals, Pfizer, Samsung Bioepis, Sandoz, UCB Pharma

DOI: 10.1136/annrheumdis-2019-eular.4421

\section{AB1280 EPIDEMIOLOGICAL PROFILE OF PATIENTS WITH FIBROMYALGIA IN A SPECIALIZED INSTITUTE IN MEDELLÍN, COLOMBIA 2010-2016}

Marcela Henao Pérez ${ }^{1}$, Diana Carolina López Medina ${ }^{1}$, Alejandra Arboleda Ramírez ${ }^{2}$, Julian Zea Osorio ${ }^{1}$, Sara Bedoya Monsalve ${ }^{1}$, Infettare. ${ }^{1}$ Universidad Cooperativa de Colombia, Medellín, Colombia; ${ }^{2}$ Instituto Neurológico de Colombia, Medellín, Colombia

Background: Fibromyalgia is a common cause of chronic musculoskeletal pain in the world, with diverse clinical manifestations and with a ruinous effect on the quality of life of patients. The estimations of prevalence in the general population are very variable $(0.2 \%-6.4 \%)$ as well as the clinical presentation.

Objectives: This study aims to describe the sociodemographic and clinical characteristics of patients with fibromyalgia treated in a specialized institution between 2010 and 2016, in Medellín, Colombia.

Methods: We performed a retrospective, descriptive, secondary source study of patients diagnosed with fibromyalgia from a specialized institution between 2010 and 2016. Descriptive statistics and period prevalence tools were applied for the variables studied, among which was evaluated the use of the diagnostic criteria (1990 or 2010) of the American College of Rheumatology.

Results: We evaluated 1106 records of patients diagnosed with fibromyalgia. The median age was 54 years (IQR 16), the age of greatest presentation is between 40 and 65 years for both sexes, $95.1 \%$ were women and a $51.1 \%$ were married or living with their partner. $23.6 \%$ of the patients had a basic and middle level education (secondary and technological level), $93 \%$ of the population came from the department of Antioquia (for a prevalence for this institution in the period 2010 to 2016 of $0.017 \%$ ), and $54 \%$ resided in the city of Medellín. The proportion of people with low and medium low socioeconomic status was $26.3 \%$, while $41.4 \%$ reported being active in a job. The symptoms most frequently presented among the patients were myalgia $(70.2 \%)$, sleep disturbances $(59.9 \%)$, chronic fatigue $(48.5 \%)$, headache $(47.3 \%)$ and muscular weakness $(40.5 \%)$. When analyzing by sex, headaches were more frequent in women $(48.8 \%)$ than in men $(24.1 \%)$. The most commonly reported comorbidities were psychiatric disorders $(31.1 \%$, where depression was the most recorded with $24.1 \%)$, migraine $(30.9 \%)$ and hypertension $(27.9 \%)$. The most used criteria to make the diagnosis of fibromyalgia were those of 1990 with $62.2 \%$. Regarding treatment, the most used were serotonin reuptake inhibitors and dual inhibitors $(52.6 \%)$, along with acetaminophen (41.2\%) and pregabalin (39\%); only $8.2 \%$ of patients received non-pharmacological interventions (physical and psychological therapy).

Conclusion: Fibromyalgia is an important pathology in specialized medical consultation, accompanied by myalgia, sleep disturbances, chronic fatigue, headaches and muscle weakness. Psychiatric pathologies are the most frequently associated comorbidities and the 2010 criteria of the American College of Rheumatology are not yet widely applied. The treatment of fibromyalgia is complex and probably more effective with a transdisciplinary approach involving both pharmacological and non-pharmacological interventions.

\section{REFERENCES}

[1] Wolfe F, Walitt B, Perrot S, Rasker JJ, Häuser W. Fibromyalgia diagnosis and biased assessment: Sex, prevalence and bias. PLoS One [Internet]. Public Library of Science; 2018 Sep 13; 13(9): e0203755.

[2] Marques AP, Santo AdSdE, Berssaneti AA, Matsutani LA, Yuan SLK Prevalence of fibromyalgia: literature review update. Revista Brasileira de Reumatologia (English Edition). 2017;57(4):356-63.

[3] Häuser W, Ablin J, Fitzcharles M-A, Littlejohn G, Luciano JV, Usui C, et al. Fibromyalgia. Nature Reviews Disease Primers. 2015;1:15022.

[4] Queiroz LP. Worldwide epidemiology of fibromyalgia. Current pain and headache reports. 2013;17(8):356.

Acknowledgement: This study was financed with funds from CONADI of the Universidad Cooperativa de Colombia. [Reference: 2042]

Disclosure of Interests: None declared

DOI: 10.1136/annrheumdis-2019-eular.1296

\section{AB1281 USAGE OF PROOF-BP TO PREDICT HYPERTENSION IN MEXICAN-MESTIZO RHEUMATIC PATIENTS}

Dionicio Ángel Galarza-Delgado ${ }^{1}$, José Ramón Azpiri-López ${ }^{2}$, Iris Jazmin ColungaPedraza $^{1}$, Carolina Marlene Martínez-Flores ${ }^{2}$, Karla Paola Cuéllar-Calderón ${ }^{1}$ Ileana Cecilia Reynosa-Silva ${ }^{1}$, Marielva Castro-González ${ }^{1}$, Raymundo Pineda ${ }^{2}$. ${ }^{1}$ Hospital Universitario, "Dr. José Eleuterio González", UANL, Rheumatology, Monterrey, Mexico; ${ }^{2}$ Hospital Universitario, "Dr. José Eleuterio González", UANL, Cardiology, Monterrey, Mexico

Background: Subjects with rheumatic diseases have an increased risk of cardiovascular morbimortality. Hypertension (HTN) is a key modifiable risk factor for cardiovascular events (1). A recently published and validated prediction model (Predicting Out-of-Office Blood Pressure, PROOF-BP) has been proposed as a tool to improve diagnosis of HTN, and detection of out-of-office HTN in subjects with a previous diagnosis, with a $c$-statistic (AUC) of 0.86 in non-rheumatic subjects (2). This model has not been explored in rheumatic patients.

Objectives: To evaluate the diagnostic performance of the PROOF-BP algorithm for the prediction of HTN in subjects with/without rheumatic diseases.

Methods: A cross-sectional, observational trial was designed. Subjects were recruited at a rheumatology outpatient clinic in northeastern Mexico. Subjects with/without rheumatologic conditions were recruited. Complete clinical history with somatometry of each subject was registered. BP was measured by a physician 3 times to each participant using current recommendations, with an OMRON HEM-7121 BP monitor. Calculations using the PROOF-BP online site were done, and risk categories were assigned to each subject using their predicted out-of-office BP: low $(<130 / 80 \mathrm{mmHg})$, medium $(130 / 80-145 / 90 \mathrm{mmHg})$ and high risk (>145/90 $\mathrm{mmHg}$ ). Subjects in the medium and high risk strata were then asked to return for further evaluation and additional BP measurement, to define each diagnosis of HTN. We used frequencies (\%) and median (q25-q75) for descriptive analysis. Diagnostic accuracy of each category was determined using $2 \times 2$ tables.

Results: A total of 217 subjects were included. Baseline characteristics are shown in Table 1. The most frequent rheumatic disease was RA $(n=78,35.9 \%)$. Using PROOF-BP, $84(38.7 \%)$ subjects were stratified as medium or high risk. Of these, only $36(42.8 \%)$ returned for evaluation A final diagnosis of HTN was attained in $14(38.8 \%)$ of those who returned. Diagnostic performances of the low and high risk categories are shown in Table 2. In $21(67.7 \%)$ cases of the medium risk category the 
diagnosis of HTN was finally discarded, and in the remaining 10 (32.2\%) diagnosis of HTN was finally ascertained. The high risk category had a specificity of $95 \%$ and a PPV of $80 \%$ for the diagnosis of HTN.

Abstract USAGE Table 1. Clinical characteristics.

\begin{tabular}{lc}
\hline & Number of patients, $\mathrm{n}(\%)$ \\
$\mathrm{N}=217$ \\
\hline Female & $188(86.6)$ \\
Hypertension & $51(23.5)$ \\
T2DM & $24(11.1)$ \\
Dyslipidemia & $68(31.3)$ \\
Active smoking & $13(6.0)$ \\
No rheumatic disease & $65(30.0)$ \\
RA & $78(35.9)$ \\
SLE & $6(2.8)$ \\
Sjögren syndrome & $5(2.3)$ \\
OA & $29(13.4)$ \\
Other & $34(15.6)$ \\
PROOF-BP class: & \\
Low risk & $133(61.3)$ \\
Medium risk & $71(33.6)$ \\
High risk & $11(5.1)$ \\
New diagnosis of hypertension & $14(38.9)$ \\
\hline
\end{tabular}

Abstract USAGE Table 2. Diagnostic performances.

\begin{tabular}{lcccc}
\hline & $\begin{array}{c}\text { Sensitivity } \\
(\%)\end{array}$ & $\begin{array}{c}\text { Specificity } \\
(\%)\end{array}$ & $\begin{array}{c}\text { PPV } \\
(\%)\end{array}$ & $\begin{array}{c}\text { NVP } \\
(\%)\end{array}$ \\
\hline Low risk & 100 & 55 & 58 & 100 \\
High risk & 29 & 95 & 80 & 68 \\
\hline
\end{tabular}

PPV: Positive predictive value; NPV: Negative predictive value.

Conclusion: In a cohort of Mexican-mestizo subjects with rheumatic diseases, $38.7 \%$ were classified as medium or high-risk for HTN. Only $42.8 \%$ of patients that required further evaluation followed medical recommendations. More than $30 \%$ of patients in the medium-risk category had a final diagnosis of HTN. Using the PROOF-BP algorithm, none of the patients in the low-risk stratum had a final diagnosis of HTN (sensitivity= $100 \%)$; the high-risk category had a high specificity (95\%) for the diagnosis of HTN.

\section{REFERENCES}

[1] Lewington S, et al. Lancet. 2002;360:1903-1913.

[2] Sheppard JP, et al. Hypertension. 2016;67:941-950.

Disclosure of Interests: None declared DOI: 10.1136/annrheumdis-2019-eular.6238

\section{AB1282 A BIG-DATA APPROACH TO ELECTRONIC HEALTH RECORD DATA - USING DIMENSIONALITY REDUCTION AND CLUSTERING TECHNIQUES TO STUDY LONGITUDINAL RELATIONSHIPS BETWEEN DISEASES}

Marc Maurits ${ }^{1}$, Thomas Huizinga ${ }^{1}$, Soumya Raychaudhuri ${ }^{2,3}$, Marcel Reinders ${ }^{4,5}$, Elizabeth Karlson ${ }^{3}$, Erik van den Akker, ${ }^{4,5}$, Rachel Knevel ${ }^{1,3}{ }^{1}{ }^{1}$ LUMC,

Rheumatology, Leiden, Netherlands; ${ }^{2}$ Brigham and Women's Hospital, Centre for Data Sciences, Boston, United States of America; ${ }^{3}$ Brigham and Women's Hospital, Rheumatology, Boston, United States of America; ${ }^{4}$ LUMC, Leiden Computational Biology Centre, Leiden, Netherlands; ${ }^{5}$ LUMC, Molecular Epidemiology, Leiden, Netherlands

Background: Hypothesis-free, longitudinal collection of patient health data in the form of Electronic Health Records (EHR) offers a wealth of valuable information on complex, slow-developing diseases in regard to aetiology and comorbidities. Conventional analytical methods are ill suited for the highly dimensional, sparse data contained within EHR, highlighting a need for more sophisticated, high-throughput tools. As t-Distributed Stochastic Neighbour Embedding (t-SNE)[1] and Density-Based Spatial Clustering of Applications with Noise (DBScan)[2] are designed to identify patterns in high-dimensional data with possible non-linear relationships, we hypothesized that these methods can aid identification of associations in diseases with multiple aetiologies.

Objectives: Proof of principle showcasing the value of t-SNE and DBScan in detecting longitudinal relationships between diseases in EHR data.
Methods: The Partners HealthCare Biobank from Boston, Massachusetts, includes 64,819 patients with longitudinal visit data from hospitals and general practitioners between June 1987 and June 2017. Each visit and procedure $(N=24,377,442)$ is coupled to an ICD code (International Classification of Disease) describing a disease or examination. We randomly split the data into two datasets of 32,424 and 32,395 individuals: set 1 to optimise t-SNE and DBScan and set 2 for replication. To trim the overly detailed hierarchy of ICDs, we translated them to Phenotype Codes (PheCodes).[3] t-SNE further reduced dimensionality and indicated groups of patients based on their PheCodes and separated patients based on PheCode patterns rather than singular codes. Subsequently DBScan identified clusters of patients in t-SNE space, by grouping patients based on relative Euclidean distance. Finally transition-probability matrices were constructed for all codes in each cluster, from which probabilistic sequences could be constructed.

We defined replication as an overlap in $\geq 25 \%$ of the PheCodes between a cluster of set 1 and 2 . Similarity was further assessed by calculating the absolute dissimilarity in transition probabilities for codes shared by matched clusters.

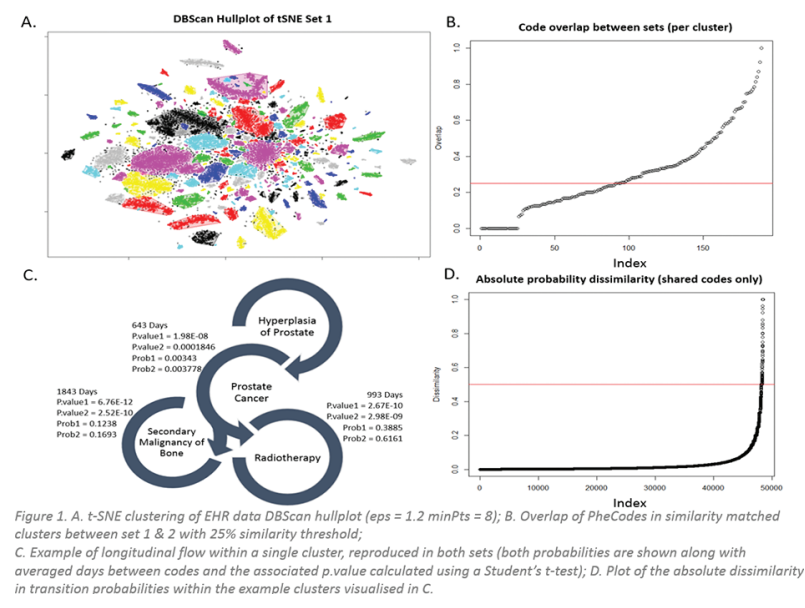

Figure 1

Results: The average (range) number of codes per individual was 376.3 $(1-8,419)$ and $375.9(1-10,315)$ spread over $4106(1-10,781)$ and $4153(1-10,746)$ days for set 1 and 2 respectively. Even though our input data was a sparse, high-dimensional $(1,865)$ matrix of PheCodes, tSNE and DBScan could clearly separate various unique patient groups with 284 and 295 clusters in set 1 and 2. Clusters consisted of patients with PheCodes of well-defined disease entities such as cardiovascular diseases and neurological disorders with objectively meaningful disease sequences. $34.5 \%$ of the clusters identified in set 1 were replicated in set 2 based on our replication criteria. Figure 1 shows the results of each step.

Conclusion: Our proof of principle supports the use of unsupervised techniques such as dimensionality reduction and data clustering to identify longitudinal associations between medical events. These methods could proof useful in our quest to identify medical risk factors for incompletely understood diseases.

\section{REFERENCES}

[1] L.J.P. v.d. Maaten, et al. Journal of Machine Learning Research 9 (Nov):2579-2605, 2008.

[2] M. Ester, et al. Proceedings of the Second International Conference on Knowledge Discovery and Data Mining: 226-231, 1996.

[3] Denny JC, et al. Bioinformatics. 2010;26(9):1205-10

Acknowledgement: Joint last authorship by R. Knevel and E.B. van den Akker. Disclosure of Interests: Marc Maurits: None declared, Thomas Huizinga Consultant for: Merck, UCB, Bristol Myers Squibb, Biotest AG, Pfizer, GSK, Novartis, Roche, Sanofi-Aventis, Abbott, Crescendo Bioscience Inc., Nycomed, Boeringher, Takeda, Zydus, Epirus, Eli Lilly, Soumya Raychaudhuri: None declared, Marcel Reinders: None declared, Elizabeth Karlson: None declared, Erik van den Akker: None declared, Rachel Knevel: None declared

DOI: 10.1136/annrheumdis-2019-eular.5281 